\title{
Glycyrrhizic acid attenuated lipid peroxidation induced by titanium dioxide nanoparticles in rat liver
}

\author{
Khorsandi $\mathrm{L}^{1,2}$, Orazizadeh $\mathrm{M}^{1}$, Mansori $\mathrm{E}^{2}$, Fakhredini $\mathrm{F}^{2}$ \\ Cell and Molecular Research Center, Faculty of Medicine, Ahvaz Jundishapur University of Medical Sciences, \\ Ahvaz, Iran.ffakhredini_aot@yahoo.com
}

\begin{abstract}
OBJECTIVE: to investigate the hepatoprotective effect of glycyrrhizic acid (GA) against hepatic injury induced by titanium dioxide nanoparticles $\left(\mathrm{NTiO}_{2}\right)$ in rats.

BACKGROUND: Many recent studies demonstrate that most nanoparticles (NPs) have an adverse or toxic action on liver.

METHODS: NTiO2- intoxicated rats received $300 \mathrm{mg} / \mathrm{kg}$ of $\mathrm{NTiO}_{2}$ for 14 days by gavage method. Protection group was pretreated with $10 \mathrm{mg} / \mathrm{kg}$ of GA for 7 days before $\mathrm{NTiO}_{2}$ administration. Alanine aminotransferase (ALT), aspartate aminotransferase (AST) and alkaline phosphatase (ALP) were detected as biomarkers in the blood to indicate hepatic injury. Product of lipid peroxidation (MDA), superoxide dismutase (SOD) and glutathione peroxidase (GPx) were evaluated for oxidative stress in hepatic injury. Light microscopy for histopathological studies was also done.

RESULTS: Administration of $\mathrm{NTiO}_{2}$ induced a significant elevation in plasma AST, ALT and ALP. In the liver, $\mathrm{NTiO}_{2}$ increased the oxidative stress through the increase in lipid peroxidation and decrease in SOD and GPX enzymes. Pretreatment of GA significantly decreased ALT, AST and ALP, attenuated the histopathology of hepatic injury, ameliorated oxidative stress in hepatic tissue, and increased the activities of SOD and GPX.

CONCLUSION: These findings indicate that GA effectively protects against $\mathrm{NTiO}_{2}$-induced hepatotoxicity in rats and might be clinically useful (Fig. 4, Ref. 47). Text in PDF www.elis.sk.

KEY WORDS: titanium dioxide nanoparticles, glycyrrhizic acid, hepatotoxicity, oxidative stress, antioxidants.
\end{abstract}

\section{Introduction}

The liver has been considered as the target organ for toxic effects of xenobiotics. The susceptibility of the liver to chemical injury is as much a function of its anatomical proximity to the bloodstream and gastrointestinal tract as to its ability to biotransform and concentrate xenobiotics (1). Previous studies have shown that administration of NPs to rodents result in their accumulation in various tissues including the liver, brain and spleen $(2,3)$.

Among the various metal nanomaterials, $\mathrm{NTiO}_{2}$ is used in a variety of consumer products such as sunscreens, cosmetics, clothing, electronics, paints, and surface coatings $(4,5)$. Recent scientific studies show that $\mathrm{NTiO}_{2}$ can be harmful to human and

${ }^{1}$ Cell and Molecular Research Center, Faculty of Medicine, Ahvaz Jundishapur University of Medical Sciences, Ahvaz, Iran, and ${ }^{2}$ Department of Anatomical Sciences, Faculty of Medicine, Ahvaz Jundishapur University of Medical Sciences, Ahvaz, Iran

Address for correspondence: F. Fakhredini, Cell and Molecular Research Center, Faculty of Medicine, Ahvaz Jundishapur University of Medical Sciences, P.O. Box 61335, Ahvaz, Iran.

Phone: +98.611.3720458, Fax: +98.611.3336380

Acknowledgement: This research was supported by a Grant (CMRC-81) from the research council of the Ahvaz Jundishapur University of Medical Sciences in 2013.

This study is part of M. SC thesis for Fereshtesadat Fakhredini and was supported by a Grant (CMRC-81) from the research council of the Ahvaz Jundishapur University of Medical Sciences in 2013. animal health (6-8). It has been reported that $\mathrm{NTiO}_{2}$ can damage liver function and induce oxidative stress and lipid peroxidation in the rodent liver (9-11).

Since NPs, such as $\mathrm{NTiO}_{2}$ affect liver function and its high rate usage it seems essential to find a suitable medication for neutralizing or decreasing their negative side effects. Herbal medicines derived from plant extracts are being increasingly utilized to treat a wide variety of clinical disease. More attention has been paid to protective effects of natural antioxidants against chemically induced toxicities $(12,13)$.

Glycyrrhizic acid (GA) is a natural constituent of liquorice isolated from the dried root of Glycyrrhiza glabra. Salts of glycyrrhizic acid are widely used as sweeteners and aromatizers in sweets, drugs, beverages, chewing-gums, chewing tobacco and toothpastes (14). It has been reported that high doses of GA can induce hypertension (15). However, GA possesses numerous pharmacological effects like anti-inflammatory, neuroprotection, anti-viral, antitumor, antioxidant (16-21) and hepatoprotective activities (21-26).

No published data were available about the daily exposure doses of $\mathrm{NTiO}_{2}$ in human. However, $\mathrm{NTiO}_{2}$ increasing use increases the health risk of people exposed to these particles, either occupationally or environmentally. Additionally, it was shown that $\mathrm{NTiO}_{2}$ accumulated mostly in the liver $(27,28)$. Thus, we used the toxic dose of $\mathrm{NTiO}_{2}$ to evaluate whether GA could prevent hepatotoxicity effects of $\mathrm{NTiO}_{2}$. 


\section{Material and methods}

Animals

In this experimental study, 32 healthy and adult male Wistar rats (8-10 weeks old, 180-200 g) were used. The animals were obtained from Ahvaz Jundishapur University of Medical Sciences, Experimental Research Center, and this study was approved by the ethics committee of Jundishapur University and carried out in an ethically proper way by following the guidelines provided. The animals were kept under standard laboratory conditions (12 h-dark and $12 \mathrm{~h}$ - light cycles, relative humidity of $50 \pm 5 \%$ and $22 \pm 3{ }^{\circ} \mathrm{C}$ ) for at least one week before the experiment and those conditions were preserved until the end of experiment. Animal cages were kept clean, and commercial food (pellet) and water were provided ad libitum.

\section{Experimental design}

The rats were randomly divided into 4 groups of 8 animals each as follows:

Group 1: Control group; received saline by gavage for 21 days Group 2: GA group; received $10 \mathrm{mg} / \mathrm{kg}$ GA by gavage for 21 days.

Group 3: $\mathrm{NTiO}_{2}$-intoxicated group; $0.2 \mathrm{ml}$ saline was administered for 7 days, and then $300 \mathrm{mg} / \mathrm{kg} \mathrm{NTiO}$, was given for 14 days.

Group4: Protection group; $10 \mathrm{mg} / \mathrm{kg}$ GA was administered for 7 days, and then GA (10 mg/kg) plus $\mathrm{NTiO}_{2}(300 \mathrm{mg} / \mathrm{kg})$ was given for 14 days.

The doses of $\mathrm{NTiO}_{2}$ (Sigma) were selected according to previous studies that demonstrated significant toxicity in rodents (29). The doses of $\mathrm{NTiO}_{2}$ (Sigma) were selected according to previous studies that demonstrated significant toxicity in rodents (29).

After characterization of $\mathrm{NTiO}_{2}$ (results not shown), the stock solution $(2 \mathrm{mg} / \mathrm{ml})$ was prepared in Milli-Q water and dispersed for $10 \mathrm{~min}$ by using a sonicator. The stock solution of $\mathrm{NTiO}_{2}$ was kept at $4{ }^{\circ} \mathrm{C}$ and used within 1 week for the experiments. Just before use, the stock solution diluted in Milli-Q water and prepared by ultrasonication (Solid State/Ultrasonic FS-14; Fisher Scientific) for $15 \mathrm{~min}$ to prevent aggregation. To ensure non-aggregation of $\mathrm{NTiO}_{2}$ before administration, the time interval from preparation to oral gavage was strictly limited in less than $20 \mathrm{~min}$. In addition, 20 min after the preparation, the particle size of $\mathrm{NTiO}_{2}$ was analyzed by atomic force microscopy (AFM).

GA (Sigma) was diluted in saline. The dose of GA was selected based on the results of previous studies $(24,30)$. One day after the last administration, after blood sampling, the rats were sacrificed by cervical dislocation under ether anesthesia and livers from each animal were removed quickly and weighed. Small pieces of liver were stored separately in a deep freezer $\left(-80{ }^{\circ} \mathrm{C}\right)$ for MDA, SOD and GPx assays. Other pieces were fixed in $10 \%$ buffered formalin.

\section{Biochemical tests}

The blood samples were collected in heparinised centrifuge tube and centrifuged. The plasma enzyme levels including plasma alanine aminotransferase (ALT), aspartate aminotransferase (AST) and alkaline phosphatase (ALP) were determined spectrophotometrically from plasma samples using commercially available kits (Sigma).

\section{Estimation of lipid peroxidation}

The degree of lipid peroxidation in liver tissue homogenate of all the experimental animals was determined in terms of thiobarbituric acid reactive substances (TBARS) formation as previously described (31). A volume of $500 \mu$ lof supernatant was mixed with $1.5 \mathrm{ml}$ trichloroacetic acid (10\%) and after centrifugation $(4,000 \times$ $\mathrm{g}$ for $10 \mathrm{~min}), 1.5 \mathrm{ml}$ of supernatant was added to $2 \mathrm{ml} \mathrm{TBA}(0.67$ $\%$ ) and heated at $100{ }^{\circ} \mathrm{C}$ for $30 \mathrm{~min}$. After cooling, the sample was extracted with $2 \mathrm{ml} \mathrm{n}$-butanol and after centrifugation at $4,000 \times \mathrm{g}$ for $15 \mathrm{~min}$, the organic phase was collected. The absorbance was read spectrophotometrically at $535 \mathrm{~nm}$. Values were expressed as $\mathrm{nmol} / \mathrm{mg}$ tissue

\section{Superoxide dismutase (SOD) activity assay}

The assay for SOD activity was made according to the method of Suttle (32) using Ransod kit (Randox Labs., Crumlin, UK). This method is based on formation of red formazan from the reaction of 2- (4-iodophenyl)-3- (4-nitrophenol)-5-phenyltetrazolium chloride and superoxide radical (produced in the incubation medium from xanthine oxidase reaction), which is assayed in a spectrophotometer at $505 \mathrm{~nm}$. The inhibition of the produced chromogen is proportional to the activity of SOD present in the sample. A 50 $\%$ inhibition is defined as 1 unit of SOD, and specific activity is expressed as units per milligram of tissue.

\section{Glutathione peroxidase (GPx) activity assay}

GPx activity was determined using the Ransel kit (Randox Labs., Crumlin, UK) according to Paglia and Valentine (1971) (33). GPx catalyzes the oxidation of glutathione by cumene hydroperoxide. In the presence of glutathione reductase and NADPH, the oxidized Glutathione (GSSG) was immediately converted to the reduced form with a concomitant oxidation of NADPH to $\mathrm{NADP}+$. The decrease in absorbance was monitored with a spectrophotometer at $340 \mathrm{~nm}$. One GPx unit is defined as $1 \mu \mathrm{mol}$ of NADPH consumed per minute, and specific activity is reported as units per milligram of tissue.

\section{Histopathology analyses.}

The formalin-fixed samples were embedded in paraffin, sectioned $(5 \mu \mathrm{m})$ and stained with haematoxylin and eosin (H\&E) for histopathology. Six stained microscopy slides per animal were examined microscopically for signs of histopathological features such as necrosis or inflammatory-cell infiltration, hepatocyte vacuolization (fatty deposits) and congestion of red blood cells (RBC).

\section{Statistical analysis}

The data were analyzed using one-way ANOVA followed by post hoc LSD test and were presented as mean \pm SD while $p<$ 0.05 was considered significant. 


\section{Results}

Nanoparticle characterizations

AFM revealed the size and morphology of the synthesized particles. The complexes appear spherical with a mean size that is inferior to $100 \mathrm{~nm}$ as can be seen in Figure 1.

\section{Biochemical tests}

GA group showed slightly lower plasma levels of ALT, AST and ALP compared to control group, but the decrease was not significant. Plasma levels of all biochemical tests were significantly increased in $\mathrm{NTiO}_{2}$ group $(\mathrm{p}<0.001)$. In $\mathrm{GA}+\mathrm{NTiO}_{2}$-treated rats, a significant reduction in the biochemical tests were observed in comparison to $\mathrm{NTiO}_{2}$-intoxicated rats $(\mathrm{p}<0.01)$. These findings are depicted in Figure 2.

\section{MDA level, SOD and GPX activities}

The administration of $\mathrm{NTiO}_{2}$ significantly increased the hepatic level of MDA when compared with the control animals. This elevation was attenuated by GA $(p<0.01)$. GPx and SOD activities were significantly decreased with $\mathrm{NTiO}_{2}$ compared to the control group ( $p<0.01$ ). Pre-administration of GA caused a significant increase in SOD and GPx when compared to $\mathrm{NTiO}_{2}$-intoxicated rats. The effects of $\mathrm{NTiO}_{2}$ and GA on MDA, SOD and GPx are reported in Figure 3.

\section{Histological analysis}

Under light microscope, liver lobular structures in control and GA groups were clear and regular, and single layer of hepatocytes was arranged around the central vein in a radial pattern (Fig. 4A). In $\mathrm{NTiO}_{2}$-intoxicated rats the normal liver lobular structures were damaged. The hepatocytes showed vacuolization and congestion of RBCs. Infiltration of inflammatory cells was also observed (Fig. 4B). These pathological changes were effectively inhibited by GA (Fig. 4C).

\section{Discussion}

Many studies have shown toxic effects of NPs but very little attention has been directed towards the neutralizing or decreasing their toxicity. To our knowledge, this is the first study that demonstrates the protective effect of a natural product against cytotoxicity induced by NPs. In this study, the protective effects of GA against $\mathrm{NTiO}_{2}$-induced hepatotoxicity were investigated. With a histopathological observation, it was possible to determine alterations in liver morphology, such as destruction of lobular structure, vacuolization of hepatocytes (fat deposits), congestion of RBC and infiltration of leukocytes in $\mathrm{NTiO}_{2}$-intoxicated rats.

Hepatocytes fatty deposits might be due to lipid peroxidation that leads to rough endoplasmic damage and detachment of the cytoplasmic lipoprotein. These findings indicate abnormal fat metabolism (34). The abnormal retention of lipids in hepatocytes induced by $\mathrm{NTiO}_{2}$ might indicate toxic injury to the liver in form of hepatocytes liposis by these particles. These results are in agreement with those of the previous investigation describing histologi-
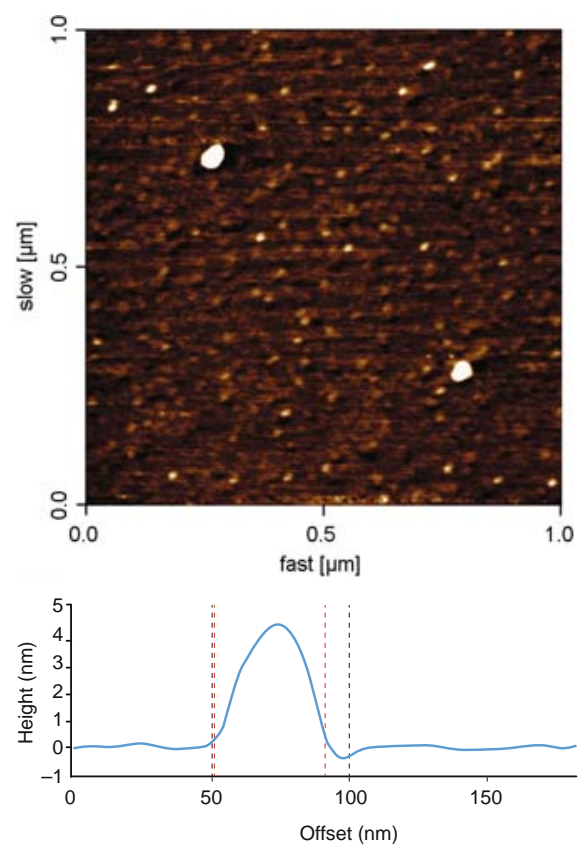

Fig. 1. AFM image of nanoparticles showed distinct spherical particles in size ranging between 50 and $100 \mathrm{~nm}$.
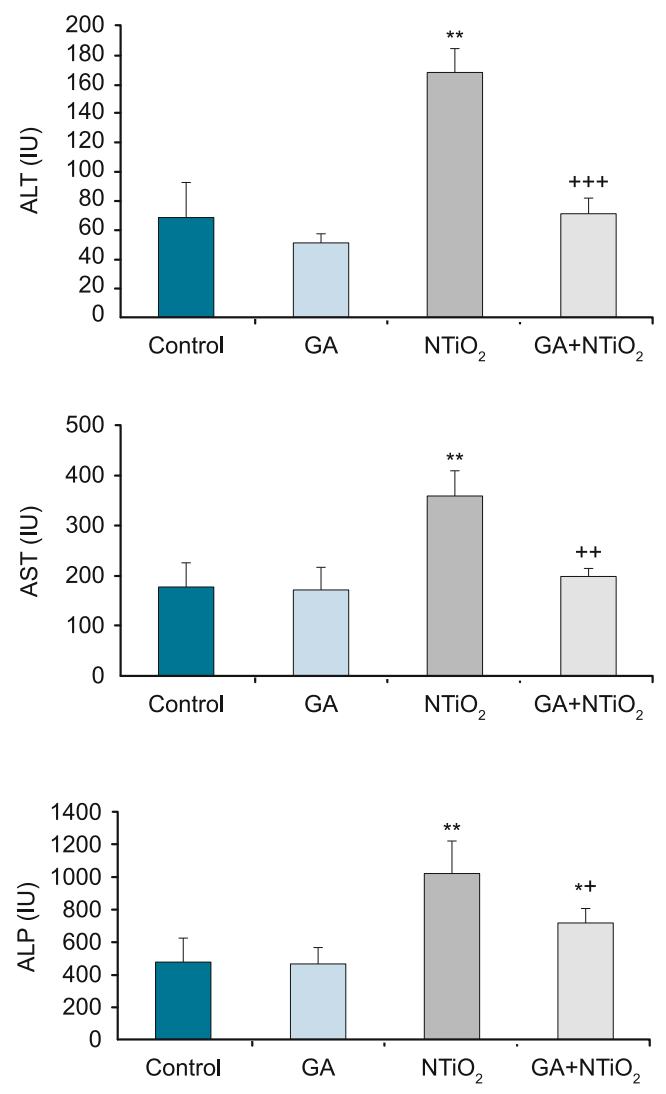

Fig. 2. Biochemical tests in control and experimental groups. Values expressed as mean \pm SD for 8 mice. ${ }^{*} p<0.01,{ }^{* *} p<0.001,{ }^{\dagger} p<0.05,{ }^{++} p$ $<0.01, "+p<0.001$; $*$ and $\dagger$ symbols respectively indicate comparison to control and $\mathrm{NTio}_{2}$-intoxicated groups. 

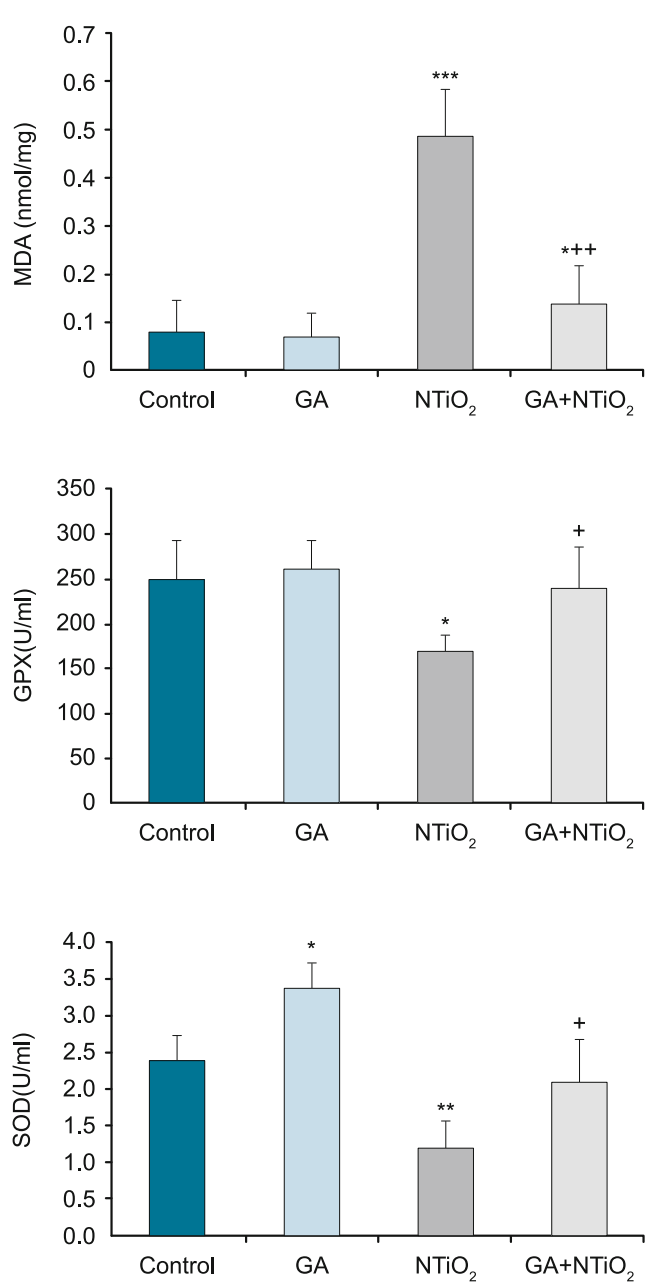

Fig. 3. MDA level, SOD and GPx activities in control and experimental groups. Values expressed as mean \pm SD for 8 mice. ${ }^{*} \mathbf{p}<0.05$, ** ${ }^{* *}<$ $0.01, * * * \mathrm{p}<0.001,{ }^{\dagger} \mathrm{p}<0.01,{ }^{+\dagger} \mathrm{p}<0.001 ; *$ and $\dagger$ symbols respectively indicate comparison to control and $\mathrm{NTio}_{2}$-intoxicated groups.

cal alterations in the liver following the administration of $\mathrm{NTiO}_{2}$. Ma et al (2009) show that $\mathrm{NTiO}_{2}$ can induce histopathological changes such as congestion of vascellum, prominent vasodilatation, vacuolization and apoptosis in liver tissue (10). In this study, the histopathological alterations were significantly attenuated by GA. GA improved lobular structure, and decreased vacuolization of hepatocytes (fat deposits), congestion of RBC and infiltration of leukocytes.

$\mathrm{NTiO}_{2}$ significantly elevated the plasma levels of ALT, AST and ALP. The plasma levels of these enzymes are the main indexes which reflect liver injury $(35,36)$. The rise in plasma AST and ALT has been attributed to the damaged structural integrity of the liver, because these are cytoplasmic in location and are released into circulation after cellular damage. ALP is localized to the bile canalicular pole of hepatocytes. In a diseased liver, this bile duct is often blocked, keeping fluid within the liver. ALP accumulates and eventually escapes into the bloodstream (35).

The reversal of alleviation of plasma enzyme activity in $\mathrm{NTiO}_{2}$ induced hepatic damage by GA could explain the prevention of leakage of intracellular enzymes by its membrane stabilizing activity.

Previous scientific researches demonstrate that $\mathrm{NTiO}_{2}$ induces oxidative stress and lipid peroxidation in the liver of rodents. Shukla et al show that $\mathrm{NTiO}_{2}$ induces oxidative DNA damage and apoptosis in human liver cells (37). The role of oxidative stress in the mechanism of NPs-induced hepatotoxicity has also been reported by Sha et al (38).

In this study, MDA concentration in liver tissue was significantly increased by $\mathrm{NTiO}_{2}$. MDA content is an index of intensified peroxidation process. GA could attenuate the $\mathrm{NTiO}_{2}$ - induced increase in the hepatic MDA content. Kiso et al demonstrate that GA can reduce lipid peroxidation (39). Wu et al also showed that 18beta-glycyrrhetinic acid prevents free fatty acid-induced hepatic lipotoxicity (40). As mentioned above histopathological analysis of our study also showed that GA effectively reduced the fatty deposits within the hepatocytes.

Oxidative stress is considered as a major risk factor that contributes to the increase in lipid peroxidation and declines the antioxidants in some degenerative diseases. Oxidative stress has been implicated as one of several mechanisms that have induced toxic effects in different organs due to enhanced production of oxygen free radicals (21).

The body has an effective defense mechanism to prevent and neutralize the free radical-induced damage. This is proficient by a set of endogenous antioxidant enzymes such as SOD and GPx.
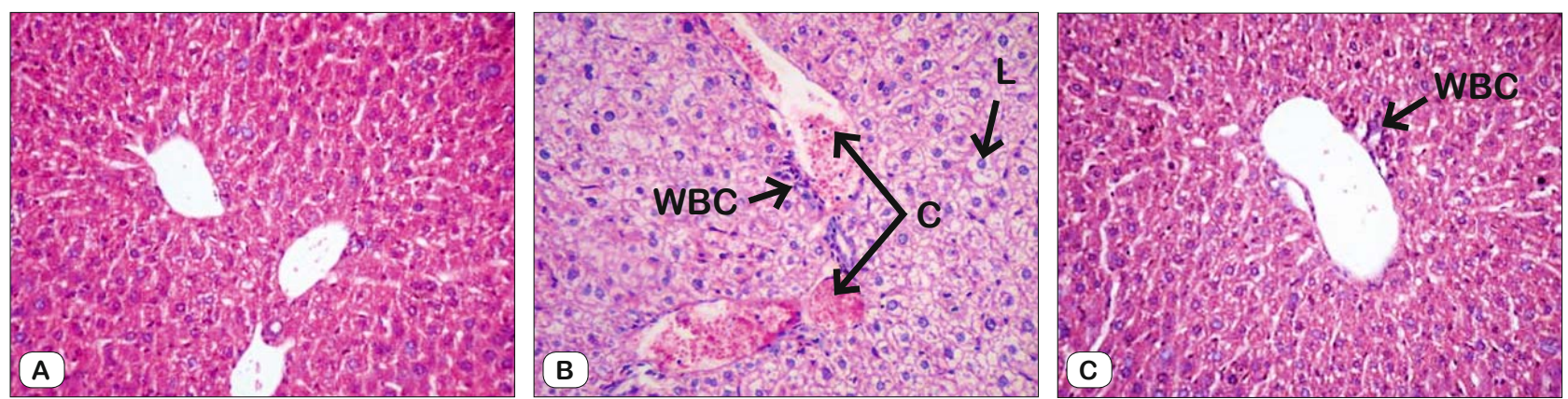

Fig. 4. Light microscopy of cross sections of H\&E-stained liver from control and experimental groups. A - Control group; B - NTio -intoxicated; C - Micro-T group. Magn. x250. 
These enzymes constitute a mutually supportive team of defense against reactive oxygen species (ROS) (41).

The observed significantly reduced activities of SOD and GPX point out the hepatic damage in the rats administered with $\mathrm{NTiO}_{2}$ but treatment with GA showed significant increase in the level of these enzymes which indicates the antioxidant activity of the GA.

Kao et al. showed that GA treatment decreased the ROS content by elevating the activities of GPx and catalase in PC12 cells (42). Rahman et al. demonstrate that GA exerts chemopreventive activity against lead acetate-induced hepatic oxidative stress (43).

Numerous scientific studies also suggest that GA has beneficial effects on the liver (22-26). Kiso et al proposed that the antioxidative action of GA plays an important role in its hepatoprotective effects against carbon tetrachloride-induced liver injury (39). Tsai et al. have been shown that GA represses total parenteral nutritionassociated acute liver injury in rats by suppressing the endoplasmic reticulum stress (26). Korenaga et al have been reported that GA-containing preparation reduces hepatic steatosis induced by hepatitis $\mathrm{C}$ virus protein and iron in mice (44).

The exact mechanism of GA protection of hepatic injury against $\mathrm{NTiO}_{2}$ is not obtained from this study. However, the reduction in AST, ALT and ALP levels indicates that GA probably reduces necrosis or apoptosis of hepatocytes. Additionally, MDA content which indicates lipid peroxidation was decreased by GA. On the other hand, GA significantly increased the antioxidant activity.

Some researchers have shown that GA induces hypertension and renal damage $(15,45)$. However, in this study, renal tissues were normal and plasma levels of BUN, $\mathrm{Cr}$ and uric acid were not significantly changed in GA $(100 \mathrm{mg} / \mathrm{kg})$ treated animals compared to control group (results not shown). Li et al have shown that pretreatment with $200 \mathrm{mg} / \mathrm{kg}$ GA improves nephrotic syndrome induced by adriamycin in rats (46). They have also reported that GA reduces the mean arterial blood pressure. Sohn et al have also demonstrated that glycyrrhizin treatment $(200 \mathrm{mg} / \mathrm{kg})$ ameliorates renal defects in rats with acute renal failure induced by gentamicin (47).

\section{Conclusion}

In conclusion, the biochemical results antioxidant enzyme assessment and histopathological findings found in the present data suggest that GA protects against $\mathrm{NTiO}_{2}$-induced hepatotoxicity. These results suggest that GA may has a potential of clinical application for treating hepatotoxicity induced by metal NPs. However, further studies will be needed to fully understand the exact mechanism of GA on NPs-induced hepatotoxicity.

\section{References}

1. Sturgill MG, Lambert GH. Xenobiotic-induced hepatotoxicity: Mechanisms of liver injury and methods of monitoring hepatic function. Clin Chem 1997; 43 (8-2): 1512-1526.

2. Borm PJ, Kreyling W. Toxicological hazards of inhaled nanoparticles potential implications for drug delivery. J Nanosci Nanotechnol 2004; 4 (5): 521-531.
3. Chen Y, Xue Z, Zheng D et al. Sodium chloride modified silica nanoparticles as a non-viral vector with a high efficiency of DNA transfer into cells. Curr Gene Ther 2003; 3 (3): 273-279.

4. Fisher J, Egerton T, Othmer K. Encyclopedia of Chemical Technology. New York, USA: John Wiley \& Sons Publisher and Distributor, 2001: 1-26.

5. Kaida T, Kobayashi K, Adachi M et al. Optical characteristics of titanium oxide interference film and the film laminated with oxides and their applications for cosmetics. J Cosmet Sci 2004; 55 (2): 219-220.

6. Oberdorster G, Oberdorster E, Oberdo rster J. Nanotoxicology: an emerging discipline evolving from studies of ultrafine particles. Environ Health Perspect 2005; 113 (7): 823-839.

7. Warheit DB, Hoke RA, Finlay C et al. Development of a base of toxicity tests using ultrafine $\mathrm{TiO} 2$ particles as a component of nanoparticle risk management. Toxicol Lett 2007; 171 (3): 99-110.

8. Ma L, Liu J, Li N et al. Oxidative stress in the brain of mice caused by translocated nanoparticulate $\mathrm{TiO} 2$ delivered to the abdominal cavity. Biomaterials 2010; 31 (1): 99-105.

9. Liu H, Ma L, Liu J et al. Toxicity of nano-anatase $\mathrm{TiO} 2$ to mice: Liver injury, oxidative stress, Toxicol. Environ Chem 2010; 92 (1): 175-186.

10. Ma $\mathbf{L}$, Zhao J, Wang $\mathbf{J}$ et al. The acute liver injury in mice caused by nano-anatase TiO2. Nanoscale Res Lett 2009; 4 (11): 1275-1285.

11. Cui Y, Gong X, Duan Y et al. Hepatocyte apoptosis and its molecular mechanisms in mice caused by titanium dioxide nanoparticles. J Hazard Mater 2010; 183 (1-3): 874-880.

12. Frei B, Higdon J. Antioxidant activity of tea polyphenols in vivo: evidence from animal studies. J Nutr 2003; 133 (10): 3275-3284.

13. Mehana EE, Meki AR, Fazili KM. Ameliorated effects of green tea extract on lead induced liver toxicity in rats. Exp Toxicol Pathol 2012; 64 (4): 291-295.

14. Fenwick G, Lutomski J, Nieman C. Liquorice, Glycyrrhiza glabra L-Composition, uses and analysis. Food Chem 1990; 38 (2): 119-143.

15. Ma SK, Bae EH, Kim IJ et al. Increased renal expression of nitric oxide synthase and atrial natriuretic peptide in rats with glycyrrhizic-acidinduced hypertension. Phytother Res 2009; 23 (2): 206-211.

16. Rackova L, Jancinova V, Petrikova $M$ et al. Mechanism of antiinflammatory action of liquorice extract and glycyrrhizin. Nat Prod Res 2007; 21 (14): 1234-1241.

17. Kim SW, Jin Y, Shin JH et al. Glycyrrhizic acid affords robust neuroprotection in the postischemic brain via antiinflammatory effect by inhibiting HMGB1 phosphorylation and secretion. Neurobiol Dis 2012; 46 (1): $147-156$.

18. Ni B, Liu Y, Zhenyu C. Glycyrrhizin protects spinal cord and reduces inflammation in spinal cord ischemia-reperfusion injury. Int J Neurosci 2013; 123 (11): 745-751.

19. Fiore C, Eisenhut M, Krausse R et al. Antiviral effects of Glycyrrhiza species. Phytother Res 2008; 22 (2): 141-148.

20. Smolarczyk R, Cichon T, Matuszczak $S$ et al. The role of glycyrrhizin, an inhibitor of HMGB1 protein, in anticancer therapy. Arch Immunol Ther Exp (Warsz) 2012; 60 (5): 391-389.

21. LiXL,ZhouAG,ZhangLet al.Antioxidant status and immune activity of glycyrrhizin in allergic rhinitis mice. Int J Mol Sci 2011; 12 (2): 905-916.

22. Wan XY, Luo M, Li XD et al. Hepatoprotective and antihepatocarcinogenic effects of glycyrrhizin and matrine. Chem Biol Interact 2009; 181 (1): 15-19. 
23. Mabuchi A, Wake K, Marlini M et al. Protection by glycyrrhizin against warm ischemia-reperfusion-induced cellular injury and derangement of the microcirculatory blood flow in the rat liver. Microcirculation 2009; 16 (4): 364-376.

24. Lee CH, Park SW, Kim YS et al. Protective mechanism of glycyrrhizin on acute liver injury induced by carbon tetrachloride in mice. Biological and pharmaceutical Bulletin 2007; 30 (10): 1898-904.

25. Guo XL, Liang B, Wang XW et al. Glycyrrhizic acid attenuates CCl4induced hepatocyte apoptosis in rats via a p53-mediated pathway. World J Gastroenterol 2013; 19 (24): 3781-3791.

26. Tsai JJ, Kuo HC, Lee KF et al. Glycyrrhizin represses total parenteral nutrition-associated acute liver injury in rats by suppressing endoplasmic reticulum stress. Int J Mol Sci 2013; 14 (6): 12563-12580.

27. Zhang R, Niu Y, Li Y et al. Acute toxicity study of the interaction between titanium dioxide nanoparticles and lead acetate in mice. Environ Toxicol Pharmacol 2010; 30 (1): 52-60.

28. Liang XJ, Chen C, Zhao Y et al. Biopharmaceutics and therapeutic potential of engineered nanomaterials. Curr Drug Metab 2008; 9 (8): 697-709.

29. Li N, Ma L, Wang $\mathrm{J}$ et al. Interaction between nano-anatase $\mathrm{TiO}_{2}$ and liver DNA from mice in vivo. Nanosacle Res Lett 2010; 5 (1): 108-115.

30. Nagai T, Egashira T, Yamanaka $Y$ et al. The protective effect of glycyrrhizin against injury of the liver caused by ischemia-reperfusion. Arch Environ Contam Toxicol 1991; 20 (3): 432-436.

31. Mansouri E, Panahi M, Ghaffari MA et al. Effects of grape seed proanthocyanidin extract on oxidative stress induced by diabetes in rat kidney. Iran Biomed J 2011; 15 (3): 100-106.

32. Suttle NF. Copper deficiency in ruminant recent developments. Vet Rec 1986; 119 (21): 519-522.

33. Paglia DE, Valentine WN. Studies on the quantitative and qualitative characterization of erythrocyte glutathione peroxidase. J Lab Clin Med 1967; 70 (1): 158-169.

34. Abdelhalim MA. Exposure to gold nanoparticles produces cardiac tissue damage that depends on the size and duration of exposure. Lipids Health Dis 2011; 10 (1): 205-214.

35. Aragon G, Younossi ZM. When and how to evaluate mildly elevated liver enzymes in apparently healthy patients. Cleve Clin J Med 2010; 77 (3): 195-204.
36. Abdel-Aziem SH, Hassan AM, Abdel-Wahhab MA. Dietary supplementation with whey protein and ginseng extract counteracts oxidative stress and DNA damage in rats fed an aflatoxin-contaminated diet. Mutat Res 2011; 723 (1): 65-71.

37. Shukla RK, Kumar A, Gurbani D et al. TiO (2) nanoparticles induce oxidative DNA damage and apoptosis in human liver cells. Nanotoxicology 2013; 7 (1): 48-60.

38. Sha B, Gao W, Wang $S$ et al. Oxidative stress increased hepatotoxicity induced by nano-titanium dioxide in BRL-3A cells and Sprague-Dawley rats. J Appl Toxicol 2014; 34 (4): 345-356.

39. Kiso Y, Tohkin M, Hikino $\mathbf{H}$ et al. Mechanism of antihepatotoxic activity of glycyrrhizin, Effect on free radical generation and lipid peroxidation. Planta Med 1984; 50 (4): 298-302.

40. Wu X, Zhang L, Gurley E et al. Prevention of free fatty acid-induced hepatic lipotoxicity by 18 beta-glycyrrhetinic acid through lysosomal and mitochondrial pathways. Hepatology 2008; 47 (6): 1905-1915.

41. Amresh G, Rao CV, Singh PN. Antioxidant activity of cissampelos pareira on benzo (a) pyrene induced mucosal injury in mice. Nutr Res 2007; 27 (10): 625-632.

42. Kao TC, Shyu M, Yen GC. Neuroprotective effects of glycyrrhizic acid and 18ß-glycyrrhetinic acid in PC12 cells via modulation of the PI3K/ Akt pathway. J Agric Food Chem 2009; 57 (2): 754-761.

43. Rahman S, Sultana S. Chemo-preventive activity of glycyrrhizin on lead acetate mediated hepatic oxidative stress and its hyper-proliferative activity in Wistar rats. Chemico Biological Interactions 2006; 160 (1): 61-69.

44. Korenaga M, Hidaka I, Nishina S et al. A glycyrrhizin-containing preparation reduces hepatic steatosis induced by hepatitis $\mathrm{C}$ virus protein and iron in mice. Liver Int 2011; 31 (4): 552-560.

45. Chubachi A, Wakui H, Asakura K et al. Acute renal failure following hypokalemic rhabdomyolysis due to chronic glycyrrhizic acid administration. Intern Med 1992; 31 (5): 708-711.

46. Li Y, Bi X, Zhu G et al. Protective effect of glycyrrhizin on nephrotic syndrome induced by adriamycin in rats. Clin Invest Med 2009; 32 (3): 229-238.

47. Sohn EJ, Kang DG, Lee HS. Protective effects of glycyrrhizin on gentamicininduced acute renal failure in rats. Pharmacol Toxicol 2003; 93 (3): 116-122. 